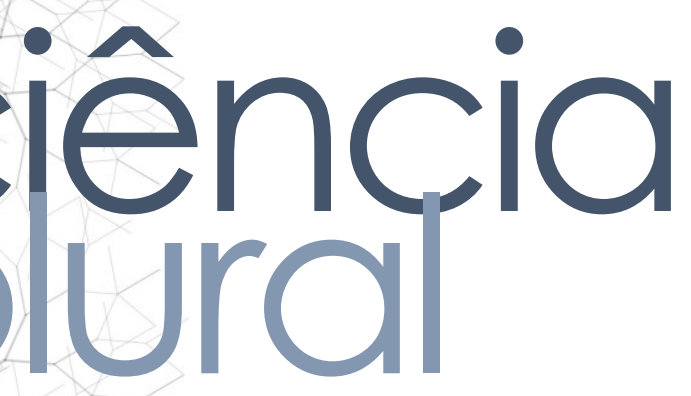

\title{
ASSISTÊNCIA AO PRÉ-NATAL NO RIO GRANDE DO NORTE: ACESSO E QUALIDADE DO CUIDADO NA ATENÇÃO BÁSICA
}

Prenatal care in Rio Grande do Norte: access and quality of care in basic care

Cuidado prenatal en Rio Grande do Norte: acceso y calidad de cuidado en cuidado básico

Cinthia Palloma Farias de Almeida • Enfermeira pela Faculdade de Ciências da Saúde do Trairí / Universidade Federal do Rio Grande do Norte - UFRN • E-mail: cinthiapfalmeida@gmail.com

Juliana Iscarlaty Freire de Araújo • Enfermeira pela Faculdade de Ciências da Saúde do Trairí / Universidade Federal do Rio Grande do Norte - UFRN • Pós-graduanda em Saúde Coletiva pela FAVENI • E-mail: ju.iscarlaty@hotmail.com

Ádilla Conceição Brito de Azevedo • Enfermeira pela Faculdade de Ciências da Saúde do Trairí / Universidade Federal do Rio Grande do Norte - UFRN • E-mail: adilla_brito@gmail.com

José Adailton da Silva • Professor adjunto • Departamento de Saúde Coletiva • Universidade Federal do Rio Grande do Norte •

E-mail: adailton.silva@ufrn.br

Autora correspondente:

Juliana Iscarlaty Freire de Araújo • E-mail: ju.iscarlaty@hotmail.com 


\section{RESUMO}

Introdução: A assistência ao pré-natal adequada e de qualidade, é primordial para redução da mortalidade materna e infantil, além disso, tem o propósito de preparar a gestante para o parto e o puerpério. Objetivo: Esta pesquisa tem como objetivo descrever a adequação da assistência pré-natal no Rio Grande do Norte, a partir dos resultados do módulo III - Entrevista com o usuário na Unidade Básica de Saúde, do Programa de Melhoria do Acesso e da Qualidade da Atenção Básica. Metodologia: Trata-se de um estudo descritivo, analítico, com abordagem quantitativa, no qual a amostra foi composta por 189 mulheres no $1^{\circ}$ Ciclo e 304 mulheres no $2^{\circ}$ Ciclo. Resultados: Os resultados obtidos foram satisfatórios para maioria das variáveis avaliadas, consideradas como essenciais pelo Ministério da Saúde quanto ao acompanhamento do pré-natal, demonstrando também, efetividade dos programas do Sistema Único de Saúde. Conclusões: Espera-se que este estudo possa incentivar estratégias no âmbito da Atenção Básica, para valorizar e ampliar os princípios do Sistema Único de Saúde, sempre trabalhando o usuário em sua completude.

Palavras-Chave: Assistência Integral à Saúde da Mulher; Atenção Primária à Saúde; Assistência Pré-Natal; Acessibilidade aos Serviços de Saúde.

\section{ABSTRACT}

Introduction: Adequate and quality prenatal care is essential to reduce maternal and child mortality, in addition, it has the purpose of preparing pregnant women for childbirth and the puerperium. Objective: This study aims to describe the adequacy of prenatal care in Rio Grande do Norte, based on the results of module III - Interview with the user in the Basic Health Unit of the Program for Improving Access and Quality of Care Basic. Methodology: This is a descriptive, analytical study with a quantitative approach, in which the sample consisted of 189 women in the 1st Cycle and 304 women in the 2 nd Cycle. Results: The results obtained were satisfactory for most of the evaluated variables, considered essential by the Ministry of Health regarding prenatal follow-up, also demonstrating the effectiveness of Health Unic System programs. Conclusions: It is hoped that this study may encourage strategies in the scope of Primary Care, to value and expand Health Unic System principles, always working the user in its completeness.

Keywords: Comprehensive Health Care for Women; Primary Health Care; Prenatal care; Health Services Accessibility..

\section{ESUMEN}

troducción: La atención prenatal adecuada y de calidad es fundamental para reducir mortalidad materna e infantil, además, tiene el propósito de preparar a las barazadas para el parto y el puerperio. Objetivo: Esta investigación tiene como tivo describir la adecuación de la asistencia prenatal en Rio Grande do Norte, a 
partir de los resultados del módulo III - Entrevista con el usuario en la Unidad Básica de Salud, del Programa de Mejora del Acceso y de la Calidad de la Atención básica. Metodología: Se trata de un estudio descriptivo, analítico, con abordaje cuantitativo, en el cual la muestra fue compuesta por 189 mujeres en el $1^{\circ}$ Ciclo y 304 mujeres en el $2^{\circ}$ Ciclo. Resultados: Los resultados obtenidos fueron satisfactorios para la mayoría de las variables evaluadas, consideradas como esenciales por el Ministerio de Salud en cuanto al acompañamiento del prenatal, demostrando también la efectividad de los programas del Sistema Único de Salud. Conclusiones: Se espera que este estudio pueda incentivar estrategias en el ámbito de la Atención Básica, para valorar y ampliar los principios del Sistema Único de Salud, siempre trabajando el usuario en su completitud.

Palabras clave: Atención Integral de Salud de la Mujer; Atención Primaria de Salud; Atención Prenatal; Accesibilidad a los Servicios de Salud. 


\section{Introdução}

A assistência ao pré-natal adequada e de qualidade é primordial para redução da mortalidade materna e infantil, pois visa ao bem-estar da gestante e seu concepto, abarcando um conjunto de cuidados e procedimentos, proporcionando a prevenção e a promoção da saúde, com identificação precoce de complicações e o tratamento favorável e adequado de doenças maternas que podem vir a surgir na gestação. Além disso, tem o propósito de preparar a gestante para o parto e o puerpérioº

Para normatização da assistência ao pré-natal no Brasil, o Ministério da Saúde (MS), em 1984, efetivou o Programa de Assistência Integral à Saúde da Mulher (PAISM) que, logo mais no ano de 2000, foi otimizado e intitulado de Programa de Humanização no Pré-Natal e Nascimento (PHPN). Dez anos depois, em 2011, nasce o Programa Rede Cegonha com o objetivo de fortalecer e validar essa política ${ }^{2}$. Concomitante a isso, o MS preconizou elementos fundamentais para a garantia de um bom acompanhamento pré-natal.

A exemplo do calendário das consultas, onde a primeira deve ser realizada o mais precoce possível, se possível até a $12^{\mathrm{a}}$ semana de gestação. A partir de então até a $28^{\mathrm{a}}$ semana as consultas são mensais, da $28^{\mathrm{a}}$ a $36^{\mathrm{a}}$ serão quinzenais e da $36^{\mathrm{a}}$ até a $41^{\mathrm{a}}$ serão semanais, de forma que ao longo de toda a gestação a mulher deve ter feito, no mínimo, seis consultas, com assistência intercalada entre enfermeiro e médico. Outra garantia, é o direito da gestante ter acesso a realização de todos exames laboratoriais necessários ${ }^{3}$.

Com a implementação destes programas, o país apontou uma queda significativa de $51 \%$ dos óbitos maternos, quando o indicador de mortalidade passou de 141 para 68 mortes para cada 100 mil nascidos vivos. Até 2015, o objetivo era diminuir o índice para 35 mortes maternas a cada 100 mil nascidos vivos 4 .

Alguns estudos mostram que a cobertura da atenção ao pré-natal nos últimos $\mathrm{z}$ anos tem aumentado em todo o Brasil, no entanto, os níveis de inadequação no tange a qualidade dos serviços ofertados ainda persistem elevados ${ }^{5}$. Segundo o em 2007 foram realizadas 12,7 milhões de consultas de pré-natal no Sistema 


\section{ciênciána
piural}

Único de Saúde (SUS) e em 2014 foram efetuadas mais de 20 milhões, o que indica um aumento positivo de $57 \%$ no número de consultas realizadas 4 .

O MS publicou a Portaria N 1.654 de 19 de julho de $2011^{6}$ que institui o Programa de Melhoria do Acesso e da Qualidade na Atenção Básica (PMAQ-AB) com o intuito de ampliar o acesso e a qualificação das práticas de gestão, cuidado e participação na Atenção Básica (AB), e além disso, com o aumento da Estratégia de Saúde da Família (ESF), que visa à reorganização da atenção básica no País, de acordo com os preceitos do SUS, alcançando em 2015 mais de 40 mil equipes e cerca de 65\% de cobertura populacional. A mesma foi revogada pela Portaria $\mathrm{N}^{\mathrm{0}} 1.6457$, de 2 de outubro de 2015, para estimular os gestores municipais do SUS a aperfeiçoar o padrão de qualidade da assistência na Atenção Primária à Saúde.

O PMAQ-AB é formado por três fases atualmente: (1) Adesão e contratualização; (2) Certificação; e (3) Recontratualização. Essas equipes são inseridas no programa pela adesão da gestão local e, após cumprimento da sua segunda fase (Certificação), que ocorre a avaliação externa, passam a ser certificadas progressivamente e contam com repasse de recursos de acordo com seu desempenho. De acordo com o Departamento de Atenção Básica (DAB), o propósito é assegurar um padrão de qualidade por meio de estratégias de qualificação, orientação e avaliação das equipes de saúde, quanto ao seu trabalho. Nessa conjuntura, um dos pontos avaliados pelo programa é a oferta de uma atenção pré-natal de qualidade ${ }^{8,9}$.

Desse modo, a cobertura da assistência ao pré-natal no Brasil vem manifestando um aumento significativo, porém, a qualidade desta ainda se apresenta abaixo do esperado, em grande parte dos munícipios brasileiros. Com isso questiona-se: Como se encontra os indicadores de qualidade e acesso à assistência pré-natal no estado do Rio Grande do Norte no contexto do PMAQ-AB?

O objetivo deste trabalho foi descrever utilizando os parâmetros do módulo III ntrevista com o usuário na Unidade Básica de Saúde, do $1^{\circ}$ e $2^{\circ}$ ciclo PMAQ- 


\section{ciêncíncia
puúl}

$\mathrm{AB}$ a adequação da assistência pré-natal no Rio Grande do Norte, no âmbito da atenção básica, conforme os índices apontados na avaliação.

\section{Metodologia}

Trata-se de um estudo descritivo, com abordagem quantitativa, pertinente ao $1^{\mathrm{o}}$ e $2^{\circ}$ ciclo da terceira fase do Programa de Melhoria do Acesso e da Qualidade da Atenção Básica (PMAQ-AB), a avaliação externa.

Os ciclos aconteceram nos anos de 2012 e 2014, respectivamente, em todos os municípios brasileiros instituídos pelo Ministério da Saúde, que aderiram voluntariamente ao PMAQ-AB, em parceria com seis instituições de pesquisa, entre elas a Universidade Federal do Rio Grande do Norte (UFRN). Para análise utilizou-se as variáveis do módulo III - Entrevista com o usuário na Unidade Básica de Saúde sobre satisfação e condições de acesso e utilização de serviços de saúde.

Delimitou-se o estudo para o Estado do Rio Grande do Norte, campo de estudo composto pelos munícipios do estado do Rio Grande do Norte que aderiram ao PMAQ-AB nos dois ciclos estudados. Segundo a Secretaria Estadual de Saúde Pública (SESAP), o estado possui 167 municípios, que são agrupados em Regiões de Saúde Pública, sendo elas: I - São José do Mipibú, II - Mossoró, III - João Câmara, IV - Caicó, V - Santa Cruz, VI - Pau dos Ferros, VII - Metropolitana e VIII - Assú, todas submetidas à SESAP10.

Os sujeitos da pesquisa foram as gestantes e puérperas, adscritas às equipes das Unidades Básicas de Saúde dos municípios que aderiram ao Programa no estado do Rio Grande do Norte no $1^{\circ}$ e $2^{\circ}$ ciclo.

Segundo estimativas do Ministério da Saúde publicadas no Portal do Departamento de Atenção Básica (DAB), 407 equipes de 112 municípios do estado articiparam dos dois momentos da pesquisa. 


\section{ciênncia
pural}

Foram excluídos do estudo os municípios que não aderiram ao programa em nenhum dos ciclos ou que participaram em apenas um dos ciclos.

No $1^{\circ}$ Ciclo, das 1.374 usuárias incluídas na avaliação, 1.162 já haviam ficado gestante alguma outra vez. Destas mulheres, 189 responderam que fizeram o pré-natal na última gestação, o que totaliza o número final de entrevistadas para a análise.

No que se refere ao $2^{\circ}$ Ciclo, das 3.045 pessoas do sexo feminino que participaram da entrevista, 2.741 afirmaram que já ficaram grávida alguma vez e destas, 304 responderam que na sua última gravidez fizeram o pré-natal na Unidade de Saúde da qual estavam sendo entrevistadas e foi esse total que foram analisadas para este ciclo.

A pesquisa foi realizada a partir da análise e descrição de dados secundários, extraídos de banco de dados disponibilizado pela UFRN, em parceria com o Ministério da Saúde sobre o $1^{\circ}$ e o $2^{\circ}$ ciclo do PMAQ-AB, a partir do módulo III - Entrevista com o usuário na Unidade Básica de Saúde sobre satisfação e condições de acesso e utilização de serviços de saúde, foram tabulados e descritos no Statistical Package for Social Sciences (SPSS) versão 20.0.

\section{Resultados}

Dos 112 municípios que aderiram ao PMAQ-AB no $1^{\circ}$ Ciclo apenas 73 municípios apresentaram mulheres que respondiam aos critérios de inclusão, a maioria das entrevistadas se concentraram nos municípios de Mossoró (7,4\%) e Natal $(6,3 \%)$ (tabela 1$)$. 
Tabela 1. Descrição da população de gestantes e puérperas, dos municípios do Rio Grande do Norte, que participaram do $1^{\circ}$ Ciclo do Programa de Melhoria do Acesso e da Qualidade (PMAQ-AB), referente ao módulo III - Entrevista com o usuário. Santa Cruz - RN, Brasil, 2018.

\begin{tabular}{lcccc}
\hline & $\mathrm{N}$ & $\%$ & Média & DP \\
\hline Idade & 142 & 74,9 & 31,38 & 70,977 \\
$21-33$ anos & & & & \\
Número de consultas & 154 & 82,5 & 7,61 & 2,116 \\
\hline $\begin{array}{l}\text { Prescrição de medicamentos preconizados } \\
\text { Sulfato Ferroso }\end{array}$ & 153 & 98,7 & 1,01 & 0,113 \\
Ácido Fólico & 150 & 96,2 & 1,04 & 0,193 \\
Realização de exames complementares & & & & \\
Urina & 153 & 98,1 & 1,02 & 0,138 \\
HIV/AIDS & 151 & 96,8 & 1,03 & 0,177 \\
VDLR & 138 & 88,5 & 1,00 & 0,321 \\
Ultrassonografia & 146 & 93,6 & 1,06 & 0,246 \\
Glicose & 142 & 91,0 & 1,09 & 0,287 \\
Orientações & & & & \\
Nutricional & 148 & 94,9 & 1,05 & 0,221 \\
Amamentação & 151 & 96,8 & 1,03 & 0,177 \\
Cuidados com a criança & 142 & 91,0 & 1,09 & 0,287 \\
Local do parto & 92 & 59,0 & 1,41 & 0,493 \\
Procedimentos & & & & \\
Medição da altura uterina & 155 & 99,4 & 1,01 & 0,080 \\
Medição da PA & 155 & 99,4 & 1,01 & 0,080 \\
Exame das mamas & 102 & 65,4 & 1,35 & 0,477 \\
Exame ginecológico & 67 & 42,9 & 1,57 & 0,497 \\
\hline
\end{tabular}

Quanto as consultas pré-natais, $47,4 \%$ tiveram consultas realizadas com o profissional médico e 52,6\% com o profissional enfermeiro. Sobre a prescrição de medicamentos preconizados pelo Ministério da Saúde, 98,7\% afirmaram que estes profissionais receitaram o Sulfato Ferroso e 96,2\% o Ácido Fólico. Para a realização de exames complementares, o exame para sífilis (VDLR) obteve a menor porcentagem entre os exames, com $88,5 \%$.

Em relação aos procedimentos executados nas consultas, percebeu-se que o ame das mamas $(65,4 \%)$, a examinação da boca $(51,3 \%)$, o exame ginecológico 
(42,9\%) e a realização do exame Papanicolau (33,3\%), atingiram menores porcentagens em relações aos demais procedimentos.

Gráfico 1. Frequência de procedimentos e orientações no acompanhamento pré-natal das gestantes e puérperas, que participaram do $1^{\circ}$ e $2^{\circ}$ Ciclo do Programa de Melhoria do Acesso e da Qualidade (PMAQ-AB), referente ao módulo III - Entrevista com o usuário. Santa Cruz - RN, Brasil, 2018.

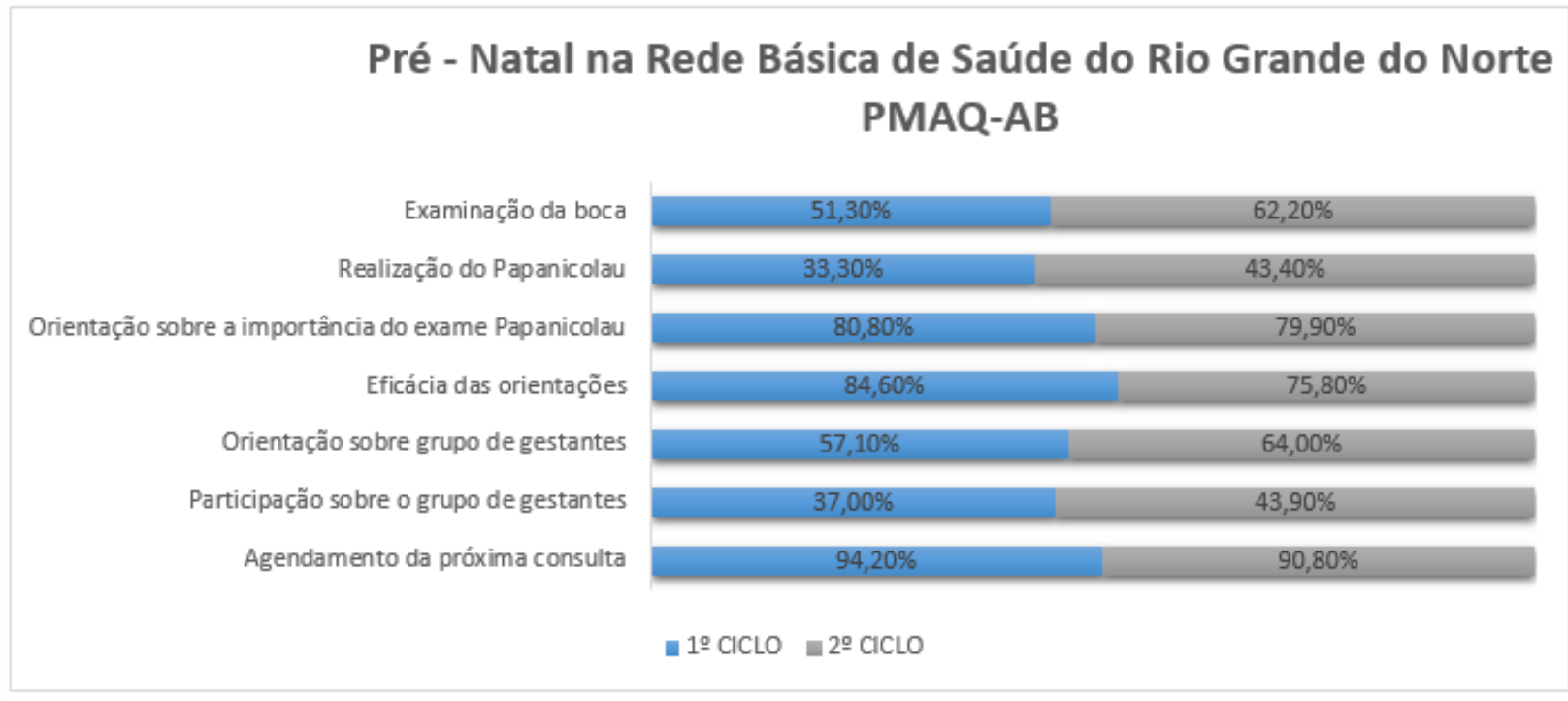

Acerca das orientações repassadas pelos profissionais durante o pré-natal, a orientação sobre o lugar (maternidade) que faria o parto foi a menos frequente, com $59 \%$.

No que se refere ao $2^{\circ}$ Ciclo (tabela 2), quando questionadas sobre quais os profissionais que realizaram as consultas, $61,5 \%$ das mulheres referiram ser acompanhada pelo médico e $94,7 \%$ pelo enfermeiro.

Tabela 2. Descrição da população de gestantes e puérperas, dos municípios do Rio Grande do Norte, que participaram do $2^{\circ}$ Ciclo do Programa de Melhoria do Acesso e da Qualidade (PMAQ-AB), referente ao módulo III - Entrevista com o usuário. Santa Cruz - RN, Brasil, 2018.

\begin{tabular}{|c|c|c|c|c|}
\hline & $\mathrm{N}$ & $\%$ & Média & DP \\
\hline \multicolumn{5}{|l|}{ Idade } \\
\hline $18-33$ anos & 256 & 84,2 & 27,02 & 5,905 \\
\hline \multicolumn{5}{|c|}{ Número de consultas } \\
\hline 6 - 10 consultas & 236 & 81,2 & 7,50 & 2,284 \\
\hline \multicolumn{5}{|c|}{ Prescrição de medicamentos preconizados } \\
\hline Sulfato Ferroso & 301 & 99,0 & 1,01 & 0,099 \\
\hline Ácido Fólico & 295 & 97,0 & 1,03 & 0,161 \\
\hline
\end{tabular}




\begin{tabular}{lllll}
\hline Realização de exames complementares & & & & \\
Urina & 301 & 99,0 & 1,01 & 0,099 \\
HIV/AIDS & 297 & 98,3 & 1,02 & 0,128 \\
VDLR & 244 & 88,4 & 1,12 & 0,321 \\
Ultrassonografia & 291 & 96,0 & 1,04 & 0,195 \\
Glicose & 277 & 92,3 & 1,08 & 0,267 \\
Orientações & & & & \\
Nutricional & 277 & 91,1 & 1,09 & 0,492 \\
Amamentação & 290 & 95,4 & 1,05 & 0,210 \\
Cuidados com a criança & 280 & 92,1 & 1,08 & 0,270 \\
Local do parto & 158 & 52,1 & 1,48 & 0,500 \\
Procedimentos & & & & \\
Medição da altura uterina & 298 & 98,0 & 1,02 & 0,139 \\
Medição da PA & 303 & 99,7 & 1,00 & 0,057 \\
Exame das mamas & 217 & 71,4 & 1,29 & 0,453 \\
Exame ginecológico & 124 & 40,8 & 1,59 & 0,492 \\
\hline
\end{tabular}

Em relação aos procedimentos que foram executados nas consultas, a inspeção das mamas (71,4\%), da boca (62,2\%), exame Papanicolau (43,4\%) e exame ginecológico $(40,8 \%)$, mostraram menor frequência quando comparado aos demais indicadores.

Acerca dos exames que as mulheres tiveram acesso durante o pré-natal, o VDLR continuou sendo o exame de menor prevalência com $88,4 \%$ e em relação as orientações, apenas 79,9\% das mulheres ouviram falar sobre a importância do exame Papanicolau e 52,1\% foram instruídas sobre o local do parto.

\section{Discussão}

Segundo o Ministério da saúde, a gestante tem direito à realização de no mínimo 6 consultas de pré-natal para uma gestação a termo, com início do acompanhamento ainda no primeiro trimestre da gestação, que devem ser executadas pelo profissional médico intercalado com o profissional de enfermagem.

Essas gestantes devem ter a garantia de acesso aos exames laboratoriais omplementares, orientações, como a alimentação, amamentação, cuidados com o RN, e alguns procedimentos essenciais ${ }^{3}$, como uma anamnese completa, seguida de me físico geral, incluindo exame ginecológico e mamário, sempre visando as cessidades de cada mulher e a idade gestacional em que ela se encontra ${ }^{10}$. 
Seguindo essa linha, o PMAQ-AB define seus indicadores e padrões de qualidade pela média de atendimentos de pré-natal por gestante cadastrada na Atenção Básica do munícipio, início do pré-natal no $1^{\circ}$ trimestre de gravidez, vacinação da gestante em dia, realização de exames citopatológicos do colo de útero e acompanhamento da gestante por meio de visitas domiciliares ${ }^{3}$. Dessa forma, a pesquisa se mostrou com resultados bastante satisfatórios com base nos indicadores supracitados, que foram destacados como essenciais pelo MS e pelo programa, para um acompanhamento pré-natal adequado e de qualidade.

Mais de $80 \%$ das mulheres entrevistadas na avaliação externa, relatam a realização de 6 ou mais consultas, em ambos os ciclos, ponto que se mostrou bastante positivo já que esse indicador afeta diretamente a qualidade e a efetividade desse acompanhamento, principalmente se aliado com uma boa assistência por parte dos profissionais. Estudos evidenciam que, quanto maior o número de consultas pré-natal, maior cobertura de vacinação antitetânica e de suplementação com sulfato ferroso, além de ocorrer redução das taxas de mortalidades neonatal e materna, redução da prevalência de prematuridade, de baixo peso ao nascer e de hipertensão arterial na gestação $^{11}$.

A prescrição de medicamentos como o sulfato ferroso e o ácido fólico também foi outro ponto bastante favorável, como preconiza o MS. O Programa Nacional de Suplementação de Ferro (PNSF), do MS, foi criado em maio de 2005, e descentralizado a partir de 2013, por meio da Portaria MS n ${ }^{0} 730$, que dispõe sobre as normas de financiamento e de execução do Componente Básico da Assistência Farmacêutica no âmbito do SUS, desde então, essa descentralização fez com que os Estados, o Distrito Federal e os Municípios fossem, a partir de então, responsáveis pela seleção, programação, aquisição, armazenamento, controle de estoque e prazos de validade, distribuição e dispensação dos medicamentos e insumos do Componente Básico da Assistência Farmacêutica, incluindo o sulfato ferroso e o ácido fólico do PNSF12.

No período gravídico, devido a uma baixa ingestão do ferro na dieta, o corpo mulher tende a ter uma maior necessidade desse nutriente, ocorrendo assim, uma 
significativa deficiência que pode vir a resultar em anemia, com frequência de 30\% entre as gestantes, segundo dados ${ }^{13}$.

Assim, o objetivo do PNSF para as gestantes é, através da suplementação preventiva de ferro e ácido fólico, além de evitar a anemiam impedir a instalação de baixos níveis de hemoglobina no momento do parto e no puerpério, e prevenir anormalidades congênitas do tubo neural do feto ${ }^{14}$. É importante que o profissional que prescreva estes e quaisquer medicamentos à paciente, tenha a disposição e o compromisso de orientar a gestante e seu(sua) acompanhante, não só da responsabilidade dos horários e frequência que ela deve se medicar, mas da importância que essa conduta dispõe para sua saúde e para a saúde do seu bebê.

A orientação atrelada à informação também se faz necessária na esfera da vacinação em qualquer circunstância, principalmente no pré-natal, sempre destacando a importância tanto para a mãe quanto para o bebê. De acordo com o calendário nacional de vacinação, a gestante deve receber as vacinas para influenza, a dupla adulto (difteria e tétano - dT), a vacina acelular contra difteria, tétano e coqueluche (dTpa) e a vacina contra hepatite B. No Módulo III do PMAQ, a variável sobre vacinação se refere apenas a vacina contra tétano, no caso, a dT e a dTpa.

O esquema da dT completo é de três doses, então no momento da $1^{\mathrm{a}}$ consulta, de preferência, o profissional deve investigar o histórico vacinal da gestante para completar ou dar continuidade ao esquema. Já a dTpa é recomendada uma dose em todas as gestações, ou seja, mulheres grávidas devem tomar uma dose da vacina em cada gestação, independente de terem tomado anteriormente. A dTpa deve ser administrada sempre a partir da $20^{\mathrm{a}}$ semana de gestação, período que gera maior proteção da criança, com efetividade estimada em 91\%. Além de proteger a gestante e evitar que ela transmita a Bordetella pertussis ao recém-nascido, permite a transferência de anticorpos ao feto, garantindo imunidade nos primeiros meses de vida até que ele complete o esquema vacinal contra coqueluche ${ }^{15}$.

Com o objetivo de coordenar essas ações de imunizações que, até então, eram efinidas pela descontinuidade de caráter episódico e limitada área de cobertura, em , foi desenvolvido o Programa Nacional de Imunizações (PNI), que objetiva a 
promoção da inclusão social ofertando assistência a todas as pessoas, em todo o país, sem discriminação de qualquer natureza, tornando-se hoje uma referência internacional de política pública de saúde.

Por meio dessas ações, o país já erradicou doenças de abrangência mundial, como a poliomielite (paralisia infantil) e a varíola. Todas as vacinas recomendadas pelas Organização Mundial da Saúde (OMS) estão à disposição de toda a população brasileira nos postos de saúde ou com equipes de vacinação, cujo dedicação e objetivo é levar a imunização até mesmo em locais de difícil acesso ${ }^{16}$.

Apesar de inúmeras dificuldades na rotina do enfermeiro no âmbito da atenção básica, no resultado das entrevistas deste estudo, a variável sobre os profissionais que realizaram as consultas das usuárias, principalmente no $2^{\circ}$ Ciclo do PMAQ, o profissional enfermeiro teve mais destaque em relação a consultas realizadas do que o profissional médico, isso nos leva a refletir que a existência da enfermagem tem se mostrado cada vez mais fundamental no cuidado na atenção básica, seja na assistência, nas práticas educativas e preventivas e/ou na gestão, sendo um trabalho estratégico e indispensável, que é reconhecido por meio dos marcos programáticos e legais do SUS.

A Lei do Exercício Profissional, $N^{\circ} 7.498 / 8617$, torna legitima a realização da Consulta de Enfermagem (CE) como atividade privativa do enfermeiro, podendo ser realizada em todos os ambientes em que ocorre o cuidado profissional de enfermagem, seja na rede pública ou privada. Popularmente, na área da saúde, muitas vezes o termo "consulta", se refere a consulta com o profissional médico, isso pode estar relacionado com a resistência inicial de alguns usuários.

A Consulta de Enfermagem é tida como um mecanismo de educação em saúde utilizada principalmente para a promoção da saúde e melhora da qualidade de vida dos usuários. Além disso, incentiva a tomada de decisões por parte do profissional baseadas em conhecimentos científicos e procedimentos que são sistematizados e valiados com frequência, desenvolvendo assim, um pensamento clínico necessário ara o cuidado de enfermagem, que relacionada à adesão de protocolos de saúde, ha possível que suas práticas assistenciais e também gerenciais, aconteçam de forma is autônoma e resolutiva ${ }^{18}$. Nesse sentido, por entender que sua assistência se torna 
mais efetiva quando integrada às demais áreas de conhecimentos e serviços que prestam amparo ao usuário do SUS, a Enfermagem tem um grande potencial de envolvimento, maior que outros profissionais, em problemas sociais da comunidade 25,19 .

Nessa perspectiva, é válido constatar que o enfermeiro deve valorizar sua autonomia, de acordo com a Lei do Exercício Profissional, fazendo bom proveito das atividades da qual possui competência legal e técnica, utilizando de bom modo a criatividade e as metodologias para incentivar essa relação com o paciente, gerando a promoção do cuidado que não seja apenas unidirecional.

Enfatizando a orientação nutricional, ponto que gerou números significativos neste estudo, quanto a informações prestadas as gestantes sobre a alimentação e ganho de peso, é importante considerar que a situação nutricional, deve ser vista como um fator determinante na qualidade da assistência prestada no pré-natal, pela sua importância no prognóstico do desenvolvimento fetal e saúde materna. Apesar da inserção do nutricionista no âmbito da atenção básica estar amparada pelos princípios do SUS e do modelo de atenção multiprofissional da ESF, há uma significativa ausência desse profissional neste espaço, fazendo com que outros profissionais, como o médico e o enfermeiro, assumam suas atribuições, de forma superficial, com conhecimentos tidos como básicos, já que não possuem formação específica e adequada. Acerca disso, existiria do mesmo modo uma melhoria na divisão do trabalho, diminuindo a sobrecarga dos demais profissionais e qualificando o atendimento ao usuário ${ }^{19}$.

Algumas variáveis deste estudo atingiram um percentual parcialmente satisfatório, de acordo com os indicadores do PMAQ e do MS. Dentre os procedimentos preconizados pelo MS, o exame da cavidade oral foi o menos realizado entre as gestantes, quanto a isso, a cobertura das equipes de saúde bucal, quando comparada com a de médicos e enfermeiros, é bem mais baixa, portanto, isso pode ser levado em consideração quando relacionamos com a escassa oferta deste exame no prế-natal na atenção básica ${ }^{8}$. 
Segundo um estudo publicado em 2015, acerca do absenteísmo em consultas odontológicas em uma Unidade Básica de Saúde de um município de grande porte, uma das conclusões da pesquisa é que o tratamento odontológico é em si, uma experiência que reflete medo, ansiedade e estresse para a maioria dos usuários e esses sentimentos apresentam ser maiores no período da gravidez, já que existe alguns mitos entre as mulheres sobre a inconveniência do tratamento odontológico durante a gestação 20 .

Diante disso, há uma necessidade clara de educação permanente em saúde, que esclareça para a população, principalmente as gestantes, quanto aos riscos e benefícios da consulta odontológica no período gravídico, esclarecendo que a saúde bucal da mãe influencia diretamente na saúde do bebê, pois problemas periodontais podem levar a um parto prematuro e nascimento de crianças abaixo do peso, por exemplo.

Para a pouca predominância na realização de exames Papanicolau, podem ter ocorrido prováveis deficiências na estrutura das Unidades Básicas de Saúde, como por exemplo, a falta de insumos e matérias necessários para a completa e segura realização desses exames ${ }^{8}$. A citologia na gravidez, é uma oportunidade para o rastreio da neoplastia do colo do útero, principalmente em mulheres que não se apresentam com regularidade ao médico para este tipo de exame. Ao contrário do que muitas mulheres podem pensar, essa análise acontece sem nenhum prejuízo para sua saúde ou a do bebê.

O pré-natal é um momento favorável para que a mulher e seu companheiro recebam orientações fundamentais, com isso os profissionais de saúde devem aproveitar bastante este período a qual encontra-se motivado e receptivo a tais informações ${ }^{21}$. Uma mulher bem informada durante o acompanhamento pré-natal, irá encarar este momento da gestação, parto e puerpério, com maior segurança, harmonia e prazer, já que a falta de orientação pode criar preocupações supérfluas e frustrações quanto as expectativas ${ }^{21}$.

A orientação quanto ao local do parto, teve sua menor frequência neste estudo, ponto importante que requer maior atenção, já que, a vinculação a uma ernidade de referência, além de ser recomendada pela Rede Cegonha² ${ }^{2}$ é direito da 


\section{ciência
piural}

gestante, garantida por lei desde 200722. Uma das finalidades dessa vinculação, é que no momento da internação para o parto, haja uma diminuição da peregrinação das mulheres na busca de uma vaga ${ }^{30}$, além de diminuir a ansiedade das futuras mães, criando uma boa oportunidade de esclarecimentos de dúvidas e inquietações, seja quanto a documentações e/ou do processo de trabalho de parto.

Em um estudo, identificou-se que $16,2 \%$ das mulheres referiram algum tipo de barreira de acesso, sendo mais recorrente em mulheres que residem no Nordeste, o que causa repercussão na deficiência do sistema de referência hospitalar para o acolhimento ao parto23, provocando possíveis riscos para a saúde do bebê e de sua mãe, principalmente a maiores episódios de óbitos neonatais e near miss materno ${ }^{24}$. É notório que uma simples conversa acerca do local, pode deixar a mulher mais confiante e tranquila no momento do parto e que esclarecimentos sobre vivências distintas devem ser trocadas frequentemente entre os profissionais de saúde e as mulheres, já que essa forma de troca de conhecimentos é considerada um importante modo de promoção da compreensão do processo de gestação.

A carência da realização de exames complementares grande parte em $100 \%$ das gestantes, retrata a perda de oportunidade de diagnosticar e tratar os danos suscetíveis de controle, como por exemplo a infecção pela sífilis, o que pode acarretar em consequências graves para o recém-nascido, tais como prematuridade, natimortalidade, manifestações congênitas precoces ou tardias e/ou morte do recémnascido ${ }^{25}$. Em ambos os ciclos do PMAQ-AB, referiu-se que a realização de VDRL foi menos frequente. Para que a sífilis congênita não venha a ocorrer, o MS recomenda que o exame deva ser realizado na primeira consulta e no terceiro trimestre do prénatal, sendo o resultado positivo, deve-se tratar imediatamente, pois o tratamento durante a gestação é benéfico tanto para a mãe quanto para o bebể

No que se refere a mudanças, como mostra o gráfico 1, há discretos avanços de um ciclo para outro, em alguns indicadores ainda não citados anteriormente, como a entação e a participação de grupo de gestantes, que merece destaque pela ampla ca de informação entre as gestantes, já que muitas vezes a troca de experiências as com as outras, se torna bem mais eficaz pela liberdade e tempo livre, do que uma 
conversa ambulatorial, mesmo que haja uma boa comunicação entre profissional e paciente. Entre esses indicadores há também as pequenas regressões, como no agendamento da próxima consulta, que está inserido na rotina de consultas da assistência pré-natal, preconizado pelo MS, importante para garantir a continuidade do cuidado e para que a gestante se sinta mais confortável em se planejar com antecedência.

Quanto a diminuição de porcentagem na eficácia das orientações, isso só reforça que a qualidade das práticas assistenciais no pré-natal, apesar de contribuir bastante, não se baseia apenas em números de consultas, por exemplo, é notável que o conteúdo dessas consultas influencia diretamente na qualidade da assistência.

\section{Conclusões}

Por meio da avaliação do PMAQ, foi possível ter conhecimento de como se encontra a qualidade do acompanhamento pré-natal no âmbito da atenção básica no Rio Grande do Norte. Sabendo que, um bom acompanhamento do pré-natal é essencial na vida de qualquer mulher, assim como na vida de seu companheiro e na de sua família, sendo um momento de grandes transformações e descobertas, originando mudanças em seus diferentes níveis, sejam eles na esfera biológica, social e /ou espiritual, qualificar o acesso à atenção primária é promover saúde visando o usuário em sua completude.

A qualidade do pré-natal não se restringe apenas a quantidade de consultas que uma gestante comparece durante sua gestação, mas como se encontra o conteúdo de cada consulta e as consequências favoráveis que esse acompanhamento gera na vida da mulher e da criança que irá nascer. Cada variável que o instrumento utilizou, como procedimentos, orientações e etc..., afeta a saúde da gestante e do seu bebê diretamente ou indiretamente, quando não colocados em práticas adequadamente ou quando essas práticas não existem na rotina do serviço.

Dado os dois ciclos avaliados, os resultados obtidos se mostraram muito sitivos, demonstrando efetividade das ações de saúde direcionadas as mulheres tantes preconizadas pelo Ministério da Saúde. Sem dúvidas, as estratégias que são 
utilizadas para promover saúde, devem estar sempre em constante renovação, para trabalhar e conhecer o usuário em diferentes perspectivas.

Valorizar programas que avaliam constantemente a qualidade e o acesso prestados ao usuário, em qualquer aspecto, como o PMAQ e estimular pesquisas nesse âmbito, é incentivar e propor estratégias, que cada vez mais enalteçam os princípios dos SUS, de acordo com a necessidade e realidade de uma certa população, além de reconhecer e auxiliar os profissionais a conduzir suas ações de acordo com as falhas expostas e analisadas.

\section{Referências}

1. Maia VKV, Lima E de FA, Costa Leite FM, Sousa AI, Primo CC. Avaliação dos indicadores de processo do Programa de Humanização no Pré-Natal e Nascimento e da Rede Cegonha. Rev Pesqui Cuid é Fundam Online [Internet]. 2017;9(4):1055.

Disponível em:

http:// seer.unirio.br/index.php/cuidadofundamental/article/view/5794

2. Ministério da Saúde. Portaria no 1.459/GM/ MS, de 24 de junho de 2011. Institui, no âmbito do Sistema Único de Saúde - SUS - a Rede Cegonha. Diário Oficial da União 2011; 23 jun.

3. Departamento de Atenção Básica, Secretaria de Atenção à Saúde, Ministério da Saúde. Saúde Mais Perto de Você. Programa Nacional da Melhoria do Acesso e da Qualidade da Atenção Básica: manual instrutivo. Brasília: Ministério da Saúde; 2012

4. Ministério da Saúde (Brasil). Ministério da Saúde libera 16 milhões para maternidade em Alagoas [publicação online]; 2016 [acesso em 05 nov 2017].

Disponível em: http:/ / portalms.saude.gov.br/noticias/agencia-saude/24044ministerio-da-saude-libera-16-milhoes-para-maternidade-em-alagoas

5. Viellas EF, Domingues RMSM, Dias MAB, Gama SGN, Theme MM Filha, da Costa JV, et al. Assistência pré-natal no Brasil. Cad Saúde Pública. 2014;30(Sup 10):S85-

S100. Disponível em:

https:/ / www.scielo.br/scielo.php?script=sci_arttext\&pid=S0102311X2014001300016.

Ministério da Saúde. Portaria no 1.645/GM/ MS, de 19 de julho de 2011. Institui, âmbito do Sistema Único de Saúde, o Programa Nacional de Melhoria do Acesso da Qualidade da Atenção Básica (PMAQ-AB) e o Incentivo Financeiro do PMAQ3, denominado Componente de Qualidade do Piso de Atenção Básica Variável B Variável. Diário Oficial da União 2011; 18 jul. 
7. Ministério da Saúde. Portaria n $1.645 / G M / M S$, de 02 de outubro de 2015. Dispõe sobre o Programa Nacional de Melhoria do Acesso e da Qualidade da Atenção Básica (PMAQ-AB). Diário Oficial da União 2015; 01 out.

8. Tomasi E, Agner P, Fernandes A, Fischer T, Carlos F, Siqueira V, et al. Qualidade da atenção pré-natal na rede básica de saúde do Brasil : indicadores e desigualdades sociais. Cad Saúde Pública; [internet] 2017;33(3):1-11. Disponível em: https:/ / www.scielo.br/pdf/csp/v33n3/1678-4464-csp-33-03-e00195815.pdf.

9. Departamento de Atenção Básica, Secretaria de Atenção à Saúde, Ministério da Saúde. Manual Instrutivo do Programa Nacional de Melhoria do Aceso e da Qualidade da Atenção Básica. Brasília: Ministério da Saúde; 2013.

10. Secretaria de Saúde Pública (Sesap). Unidades Regionais de Saúde. Rio Grande do Norte; [publicação online]; 2017 [acesso em 10 abr 2018]. Disponível em: http:/ / www.saude.rn.gov.br/Conteudo.asp?TRAN=ITEM\&TARG=7556\&ACT=\&P $\mathrm{AGE}=0 \& \mathrm{PARM}=\& \mathrm{LBL}=\mathrm{URSAP}$.

11. Secretaria de Saúde. Coordenadoria de Planejamento em Saúde. Assessoria Técnica em Saúde. Atenção à gestante e à puérpera no SUS - SP: manual técnico do pré natal e puerpério. Estado de São Paulo; [publicação online] 2010 [acesso em 09 nov 2018]. Available from:

http://www.saude.sp.gov.br/resources/ses/perfil/gestor/destaques/atencao-agestante-e-a-puerpera-no-sus-sp/manual-tecnico-do-pre-natal-epuerperio/manual_tecnicoii.pdf.

12. Ministério da Saúde. Portaria n ${ }^{\circ}$ 730/GM/ MS, de 13 de maio de 2005. Institui o Programa Nacional de Suplementação de Ferro, destinado a prevenir a anemia ferropriva e dá outras providências. Diário Oficial da União 2005; 12 mai.

13. Ministério da Saúde. Portaria nº 1.555/GM/ MS, de 30 de julho de 2013. Dispõe sobre as normas de financiamento e de execução do Componente Básico da Assistência Farmacêutica no âmbito do Sistema Único de Saúde (SUS). Diário Oficial da União 2013; 29 jul.

14. Lunardi-Maia T, Schuelter-Trevisol F, Galato D. Uso de medicamentos no primeiro trimestre de gravidez : avaliação da segurança dos medicamentos e uso de ácido fólico e sulfato ferroso. Rev Bras Ginecol e Obs; [internet] 2014;36(12):541-7. Disponível em: https:// www.scielo.br/scielo.php?pid=S010072032014001200541\&script=sci_abstract\&tlng=pt.

Sociedade Brasileira de Imunizações, Ministério da Saúde. Calendário de cinação da Gestante. Brasília: Ministério da Saúde; 2018.

Ministério da Saúde (Brasil), Programa Nacional de Imunizações (PNI); 2015. 
17. Ministério da Saúde. Lei no 7.498/GM/ MS, de 27 de junho de 1986. Dispõe sobre a regulamentação do exercício da enfermagem, e dá outras providências. Diário Oficial da União 1986; 26 jun.

18. Kahl C, Meirelles BHS, Lanzoni GM de M, Koerich C, Cunha KS da. Ações e interações na prática clínica do enfermeiro na Atenção Primária à Saúde. Rev Esc Enferm USP; [internet] 2018;24(4):1-7. Disponível em: https:/ / www.scielo.br/pdf/reeusp/v52/0080-6234-reeusp-52-e03327.pdf.

19. Rosane C, Arruda P. O nutricionista na Atenção Básica em saúde : uma revisão de literatura. Mestr em Atenção Integr à Saúde Brasília: Universidade de Brasília Faculdade de Ciências da Saúde, 2016.

20. Pereira AC, Luciana K, Sarracini M. Estratégias para o enfrentamento do absenteísmo em consultas odontológicas nas Unidades de Saúde da Família de um município de grande porte : uma pesquisa-ação. Cien Saude Colet; [internet] 2015;20(2):449-60.Disponível em: https:// www.scielo.br/scielo.php?pid=S1413$81232015000200449 \&$ script=sci_arttext.

21. Rodrigues AP, Maris S, Padoin DM, Guido LDA, Felipe L, Lopes D. Fatores do pré-natal e do puerpério que interferem na autoeficácia em amamentação. Esc Anna Nery; [internet] 2014;18(2):257-61. Disponível em: https:/ / www.scielo.br/scielo.php?script=sci_abstract\&pid=S141481452014000200257\&lng=en\&nrm=iso\&tlng=pt.

22.Ministério da Saúde. Lei $n^{\circ}$ 11.634/GM/ MS, de 27 de dezembro de 2007. Dispõe sobre o direito da gestante ao conhecimento e a vinculação à maternidade onde receberá assistência no âmbito do Sistema Único de Saúde. Diário Oficial da União 2007; 28 dez.

23. Viellas EF, Augusto M, Dias B, Viana J, Bastos MH. Assistência pré-natal no Brasil. Cad Saúde Pública; [internet] 2014;30(1):85-100. Disponível em: https:/ / www.scielo.br/scielo.php?script=sci_arttext\&pid=S0102311X2014001300016.

24. Lansky S, Friche AA de L, Silva AAM da, Campos D, Bittencourt SD de A, Carvalho ML de, et al. Pesquisa Nascer no Brasil : perfil da mortalidade neonatal e avaliação da assistência à gestante e ao recém-nascido. Cad Saúde Pública; [internet] 2014;30(1):192-207. Disponível em: https:// www.scielo.br/pdf/csp/v30s1/0102311X-csp-30-s1-0192.pdf.

25. Brasil. Ministério da Saúde. Secretaria de Vigilância em Saúde. Departamento de gilância, Prevenção e Controle das IST, do HIV / Aids e das Hepatites Virais. rotocolo Clínico e Diretrizes Terapêuticas para Atenção Integral às Pessoas com fecções Sexualmente Transmissíveis. Brasília, DF; 2018. 